\title{
Generation and morphing of plasmons in graphene superlattices
}

\author{
Andrea Tomadin, ${ }^{1, *}$ Francisco Guinea, ${ }^{2}$ and Marco Polini ${ }^{1,3}$ \\ ${ }^{1}$ NEST, Istituto Nanoscienze-CNR and Scuola Normale Superiore, I-56126 Pisa, Italy \\ ${ }^{2}$ Instituto de Ciencia de Materiales de Madrid (CSIC), Sor Juana Inés de la Cruz 3, E-28049 Madrid, Spain \\ ${ }^{3}$ Istituto Italiano di Tecnologia, Graphene Labs, Via Morego 30, I-16163 Genova, Italy
}

(Received 17 July 2014; published 23 October 2014)

\begin{abstract}
Recent experimental studies on graphene on hexagonal boron nitride $(\mathrm{hBN})$ have demonstrated that $\mathrm{hBN}$ is not only a passive substrate that ensures superb electronic properties of graphene's carriers, but that it actively modifies their massless Dirac fermion character through a periodic moiré potential. Here we present a theory of the plasmon excitation spectrum of massless Dirac fermions in a moiré superlattice. We demonstrate that graphene-hBN stacks offer a rich platform for plasmonics in which control of plasmon modes can occur not only via electrostatic gating but also by adjusting, e.g., the relative crystallographic alignment.
\end{abstract}

DOI: 10.1103/PhysRevB.90.161406

PACS number(s): 73.20.Mf, 42.30.Ms, 78.67.Wj

Introduction. Vertical heterostructures [1-3] comprising graphene and two-dimensional (2D) hexagonal boron nitride (hBN) crystals [4] offer novel opportunities for applications [5] and fundamental studies of electron-electron interactions [6-8]. Recent experimental studies [9-12] have demonstrated that hBN substantially alters the electronic spectrum of the massless Dirac fermion (MDF) carriers hosted in a nearby graphene sheet. Indeed, when graphene is deposited on hBN, it displays a moiré pattern [13,14], a modified tunneling density of states [9], and self-similar transport characteristics in a magnetic field [10-12]. The potential produced by $\mathrm{hBN}$ acts on graphene's carriers as a perturbation with the periodicity of the moiré pattern $[15,16]$. This is responsible for a reconstruction of the MDF spectrum and the emergence of minibands in the moiré superlattice Brillouin zone (SBZ) $[15,16]$.

A parallel line of research has focused a great deal of attention on graphene plasmonics [17]. Here, the goal is to exploit the interaction of infrared light with "Dirac plasmons" (DPs) - the self-sustained density oscillations of the MDF liquid in a doped graphene sheet [18] — for a variety of applications such as infrared [19] and terahertz [20] photodetectors. Interest in graphene plasmonics considerably increased after two experimental groups [21,22] showed that the DP wavelength is much smaller than the illumination wavelength, allowing an extreme concentration of electromagnetic energy, and easily gate tunable. These experiments were not optimized to minimize DP losses. Microscopic calculations $[23,24]$ indicate that these can be strongly reduced by using $\mathrm{hBN}$ (rather than, e.g., $\mathrm{SiO}_{2}$ ) as a substrate.

In this Rapid Communication we try and combine the ideas of these two fields of research by presenting a theory of the impact of a moiré superlattice on graphene's plasmons. Following recent transport experiments [10-12], we focus our attention on the case of long-wavelength superlattices since, in this case, interesting features in the miniband structure appear at carrier densities that can be achieved by standard electrostatic gating. By employing linear response theory within the random phase approximation [25], we calculate the plasmon modes of 2D MDFs in a moiré superlattice. We have found that graphene-hBN superlattices harbor a wealth of satellite plasmons. These modes emerge from electron-hole pockets located at the high-symmetry, e.g., $\tilde{M}, \tilde{K}$, and $\tilde{K}^{\prime}$, points in the SBZ-see Fig. 1(a). Depending on the nature of the moiré superlattice, the $\tilde{\Gamma}$-point plasmon, which at low doping is an ordinary DP mode [17], may survive or morph into a satellite plasmon when the chemical potential increases. The concept of plasmon morphing is sketched in Fig. 1(b). Spectroscopy of plasmons in graphene superlattices therefore reveals precious information on the properties of the miniband structure. Even more interestingly, graphene-hBN stacks offer a low-loss plasmonic platform where knobs other than electrostatic gating [17]—-such as the twist angle between the graphene and hBN crystals - can be used for the manipulation of plasmons.

Moiré superlattice minibands. We describe the problem of a MDF moving in a moiré superlattice with the following single-particle continuum-model Hamiltonian [16],

$\mathcal{H}_{0}=\hbar v_{\mathrm{F}} \boldsymbol{\sigma} \cdot \boldsymbol{p} \tau_{0}+\hbar v_{\mathrm{F}} \boldsymbol{\sigma} \cdot \boldsymbol{A}(\boldsymbol{r}) \tau_{3}+V(\boldsymbol{r}) \sigma_{0} \tau_{0}+\Delta(\boldsymbol{r}) \sigma_{3} \tau_{3}$,

acting on the four-component pseudospinor $\left(\Psi_{A, K}, \Psi_{B, K}\right.$, $\left.\Psi_{B, K^{\prime}},-\Psi_{A, K^{\prime}}\right)^{\mathrm{T}}$. In Eq. (1), $\boldsymbol{p}=-i \hbar \nabla_{\boldsymbol{r}}$ is the 2D momentum measured from the centers of the two graphene's principal valleys $v=K, K^{\prime}, v_{\mathrm{F}} \sim 10^{6} \mathrm{~m} / \mathrm{s}$ is the Fermi velocity in an isolated graphene sheet, and $\sigma_{a}, \tau_{a}$ with $a=0,1,2,3$ are ordinary $2 \times 2$ Pauli matrices acting on graphene's sublattice and principal-valley degrees of freedom, respectively $\left(\sigma_{0}\right.$ and $\tau_{0}$ are identity matrices). Finally, $V(\boldsymbol{r}), \boldsymbol{A}(\boldsymbol{r})$, and $\Delta(\boldsymbol{r})$ are potentials due to the moiré superlattice. Note that the terms containing $\boldsymbol{A}$ and $\Delta$ have different signs in the two principal valleys. Since $V(\boldsymbol{r}), \boldsymbol{A}(\boldsymbol{r})$, and $\Delta(\boldsymbol{r})$ are periodic perturbations, we can expand them in the following form: $V(\boldsymbol{r})=$ $\sum_{\boldsymbol{G}} V(\boldsymbol{G}) \exp (i \boldsymbol{G} \cdot \boldsymbol{r}), \quad \boldsymbol{A}(\boldsymbol{r})=\sum_{\boldsymbol{G}} \boldsymbol{A}(\boldsymbol{G}) \exp (i \boldsymbol{G} \cdot \boldsymbol{r})$, and $\Delta(\boldsymbol{r})=\sum_{\boldsymbol{G}} \Delta(\boldsymbol{G}) \exp (i \boldsymbol{G} \cdot \boldsymbol{r})$, where $\boldsymbol{G}$ denotes the reciprocal lattice vectors (RLVs) of the moiré superlattice. Since $\mathcal{H}_{0}$ is block diagonal in valley space, one can find the spectrum of $\mathcal{H}_{0}$ in each principal valley $v=K, K^{\prime}$, separately.

The eigenvectors $|\boldsymbol{k}, n, v\rangle$ of $\mathcal{H}_{0}$ can be expanded in a planewave basis as

$$
\langle\boldsymbol{r} \mid \boldsymbol{k}, n, v\rangle=\frac{1}{\sqrt{L^{2}}} \sum_{\boldsymbol{G}} \boldsymbol{u}_{\boldsymbol{G}}(\boldsymbol{k}, n, v) e^{i(\boldsymbol{k}+\boldsymbol{G}) \cdot \boldsymbol{r}},
$$



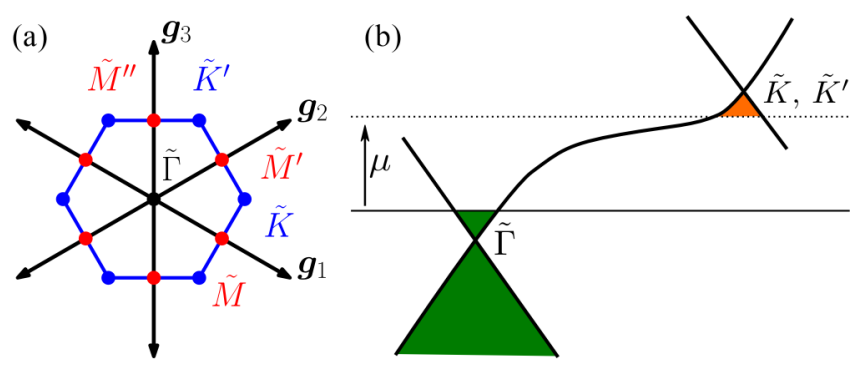

FIG. 1. (Color online) (a) shows the SBZ with its high-symmetry points and the first star of reciprocal lattice vectors $\pm \boldsymbol{g}_{1}, \pm \boldsymbol{g}_{2}$, and $\pm \boldsymbol{g}_{3}$. Here, the $\tilde{\Gamma}$ point (i.e., the center of the SBZ) coincides with the $v=K, K^{\prime}$ points of the original graphene's Brillouin zone. (b) illustrates the concept of plasmon morphing. Two Dirac-band crossings are located at different points of the SBZ and are separated in energy. When the chemical potential $\mu$ lies at the position of the horizontal solid line, the system displays the plasmon $[17,18]$ of a 2D Dirac system with $n$-type doping (green shaded area). Upon increasing the chemical potential (dashed line), the $\tilde{\Gamma}$-point plasmon morphs into the $\tilde{K} / \tilde{K}^{\prime}$-point plasmon of a $2 \mathrm{D}$ Dirac system with $p$-type doping (orange shaded area).

where $L^{2}$ is the $2 \mathrm{D}$ electron system area and $\sum_{\boldsymbol{G}} \boldsymbol{u}_{\boldsymbol{G}}\left(\boldsymbol{k}, n^{\prime}, v^{\prime}\right)^{\dagger} \boldsymbol{u}_{\boldsymbol{G}}(\boldsymbol{k}, n, v)=\delta_{n, n^{\prime}} \delta_{v, v^{\prime}}$. The wave vector $\boldsymbol{k}$ varies in the first SBZ, $n$ is a discrete index for the superlattice minibands, and $v=K, K^{\prime}$. Eigenvalues $\varepsilon_{\boldsymbol{k}, n, v}$ and eigenvector components $\boldsymbol{u}_{\boldsymbol{G}}(\boldsymbol{k}, n, v)$ can be found by solving the secular equation [26]

$$
\sum_{\boldsymbol{G}^{\prime}} \mathcal{H}_{\boldsymbol{G}, \boldsymbol{G}^{\prime}}(\boldsymbol{k}) \boldsymbol{u}_{\boldsymbol{G}^{\prime}}(\boldsymbol{k}, n, v)=\varepsilon_{\boldsymbol{k}, n, v} \boldsymbol{u}_{\boldsymbol{G}}(\boldsymbol{k}, n, v),
$$

where $\quad \mathcal{H}_{\boldsymbol{G}, \boldsymbol{G}^{\prime}}(\boldsymbol{k}) \equiv \hbar v_{\mathrm{F}} \boldsymbol{\sigma} \cdot(\boldsymbol{k}+\boldsymbol{G}) \delta_{\boldsymbol{G}, \boldsymbol{G}^{\prime}} \tau_{0}+\hbar v_{\mathrm{F}} \boldsymbol{\sigma} \cdot \boldsymbol{A}(\boldsymbol{G}-$ $\left.\boldsymbol{G}^{\prime}\right) \tau_{3}+V\left(\boldsymbol{G}-\boldsymbol{G}^{\prime}\right) \sigma_{0} \tau_{0}+\Delta\left(\boldsymbol{G}-\boldsymbol{G}^{\prime}\right) \sigma_{3} \tau_{3}$. The size of the matrix $\mathcal{H}_{\boldsymbol{G}, \boldsymbol{G}^{\prime}}(\boldsymbol{k})$ is $4 \mathcal{N} \times 4 \mathcal{N}$, where $\mathcal{N}$ is the number of RLVs included in the expansion in Eq. (2). The miniband index $n$ is chosen such that $\varepsilon_{\boldsymbol{k}, n, v} \leqslant \varepsilon_{\boldsymbol{k}, n+1, v}$, with $\varepsilon_{\tilde{\boldsymbol{K}},-1, v}<0<\varepsilon_{\tilde{\boldsymbol{K}}, 1, v}$. The dispersion of the first conduction (valence) miniband is thus $\varepsilon_{\boldsymbol{k}, 1, v}\left(\varepsilon_{\boldsymbol{k},-1, v}\right)$.

The sparseness of $\mathcal{H}_{\boldsymbol{G}, \boldsymbol{G}^{\prime}}(\boldsymbol{k})$ is controlled by the number of RLVs included in the Fourier representation of the moiré potentials $V, \boldsymbol{A}$, and $\Delta$. Following Refs. [15,16], we use only six RLVs $\pm g_{1}, \pm g_{2}$, and $\pm g_{3}$ in the Fourier expansion of the moiré potentials. For two twisted honeycomb lattices with lattice constants $a$ and $a(1+\delta)$, the RLV $g_{1}$ is given by [9] $g_{1}=4 \pi(\cos (\theta), \sin (\theta)) /(\sqrt{3} \lambda)$, where $\lambda=$ $(1+\delta) a / \sqrt{2(1+\delta)[1-\cos (\phi)]+\delta^{2}}$ is the moire superlattice wavelength and $\phi$ the relative rotation angle between the two honeycomb lattices. Finally, $\theta$ is the relative rotation angle of the moiré pattern with respect to the graphene lattice with $\tan (\theta)=\sin (\phi) /[(1+\delta)-\cos (\phi)]$. The RLV $\boldsymbol{g}_{2}\left(\boldsymbol{g}_{3}\right)$ can be obtained from $g_{1}$ by a counterclockwise rotation of $\pi / 3(2 \pi / 3)$, as shown in Fig. 1(a). For graphene on hBN, $a=\sqrt{3} a_{0}$ is the graphene lattice constant $\left(a_{0} \simeq 1.42 \AA\right)$ and $\delta \simeq 1.8 \%$.

For the sake of simplicity, we focus our attention on the case in which pseudomagnetic fields are absent, i.e., $\boldsymbol{A}(\boldsymbol{r})=\mathbf{0}$, although the theoretical apparatus described below is completely general. We then write the scalar potential as $V(\boldsymbol{r})=2 V_{\mathrm{s}} \sum_{m=1 \ldots 3} \cos \left(\boldsymbol{g}_{m} \cdot \boldsymbol{r}\right)$ and the mass term as $\Delta(\boldsymbol{r})=$

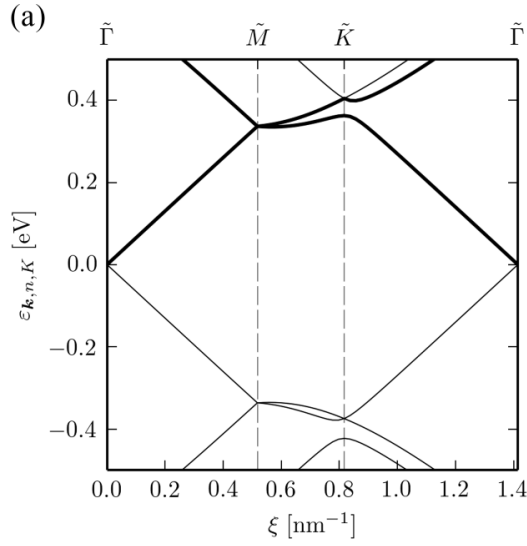

(b)
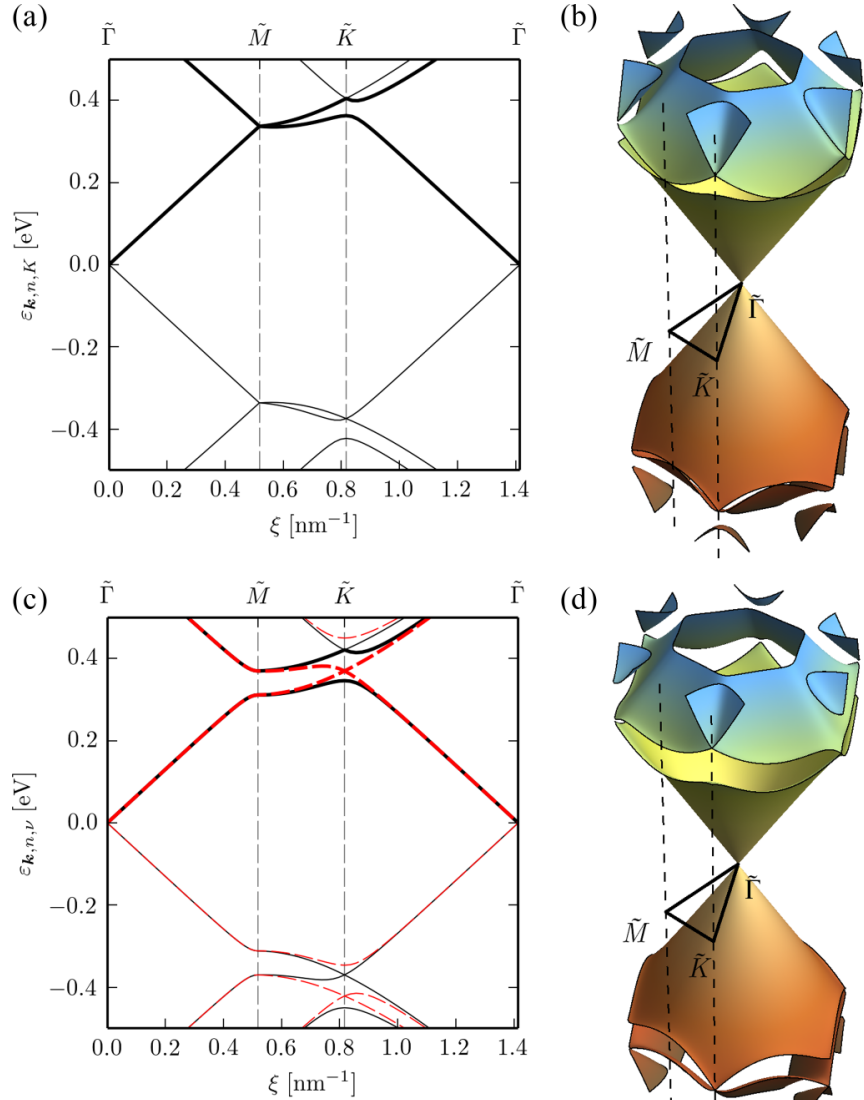

(d)

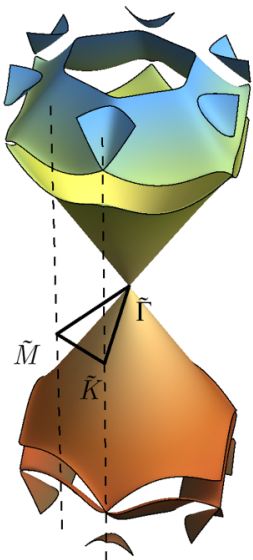

FIG. 2. (Color online) Graphene-hBN superlattice minibands along the $\tilde{\Gamma}-\tilde{M}-\tilde{K}-\tilde{\Gamma}$ direction (twist angle $\phi \simeq 0.03 \mathrm{rad}, \lambda \simeq 7 \mathrm{~nm}$ ). Thick lines denote the first two conduction minibands. All the numerical results in this figure have been obtained by including $\mathcal{N}=37$ RLVs $\left(|\boldsymbol{G}| \leqslant 3\left|\boldsymbol{g}_{1}\right|\right)$ in Eq. (2). (a) refers to a moiré potential with $V_{\mathrm{s}}=30 \mathrm{meV}$ and $V_{\Delta}=0$. (b) gives a $3 \mathrm{D}$ representation of the moiré superlattice miniband structure for the same parameters as in (a). (c) and (d) refer to a moiré potential with $V_{\mathrm{s}}=0$ and $V_{\Delta}=30 \mathrm{meV}$. In (c) black solid (red dashed) lines label the miniband structure around the $K\left(K^{\prime}\right)$ principal valley.

$-2 V_{\Delta} \sum_{m=1 \ldots 3} \sin \left(\boldsymbol{g}_{m} \cdot \boldsymbol{r}\right)$, implying that the Hamiltonian $\mathcal{H}_{0}$ is inversion symmetric [27]. Illustrative numerical results for the minibands of a long-wavelength $(\phi \ll 1)$ graphene-hBN moiré superlattice are reported in Fig. 2. We focus our attention on the features of the miniband structure occurring in the first two conduction minibands-the thick lines in Figs. 2(a) and 2(c). Features at these energies can be accessed via electrostatic doping.

For $V_{\mathrm{s}} \neq 0$ and $V_{\Delta}=0$ [Fig. 2(a)], we see that the spectrum hosts a crossing at the $\tilde{M}$ point of the SBZ. This crossing evolves into an isolated Dirac point for scalar potentials with larger amplitude, e.g., $V_{\mathrm{s}} \sim 100 \mathrm{meV}$. Since experiments [9-12] seem to indicate weaker potentials, we have decided to use $V_{\mathrm{s}}=30 \mathrm{meV}$. Moreover, plasmons emerging from an isolated Dirac point have well studied properties [17]. For $V_{\mathrm{s}}=0$ and $V_{\Delta} \neq 0$ [Fig. 2(c)], the spectrum shows satellite Dirac points at the $\tilde{K}$ (in one principal valley) and $\tilde{K}^{\prime}$ (in the other principal valley) points of the SBZ. See Ref. [28] for further important considerations on Fig. 2(c). 
Plasmons in a moiré superlattice. Complete information on the plasmon modes of an interacting system of MDFs in a moiré superlattice is contained in the density response function [25] $\chi_{\boldsymbol{G}, \boldsymbol{G}^{\prime}}(\boldsymbol{q}, \omega) \equiv \chi_{n n}\left(\boldsymbol{q}+\boldsymbol{G}, \boldsymbol{q}+\boldsymbol{G}^{\prime}, \omega\right)$, viewed as a matrix with respect to the RLVs $\boldsymbol{G}, \boldsymbol{G}^{\prime}$ and with $\boldsymbol{q}$ spanning the first SBZ. We also introduce the inverse dielectric matrix [25],

$$
\left[\epsilon^{-1}\right]_{\boldsymbol{G}, \boldsymbol{G}^{\prime}}(\boldsymbol{q}, \omega)=\delta_{\boldsymbol{G}, \boldsymbol{G}^{\prime}}+v_{\boldsymbol{G}}(\boldsymbol{q}) \chi_{\boldsymbol{G}, \boldsymbol{G}^{\prime}}(\boldsymbol{q}, \omega),
$$

where $v_{\boldsymbol{G}}(\boldsymbol{q})=v(\boldsymbol{q}+\boldsymbol{G})$ with $v(q)=2 \pi e^{2} /(\epsilon q)$ the 2D Fourier transform of the Coulomb potential. Here $\epsilon=\left(\epsilon_{1}+\right.$ $\left.\epsilon_{2}\right) / 2$ is the average of the dielectric constants of the media above $\left(\epsilon_{1}\right)$ and below $\left(\epsilon_{2}\right)$ the graphene flake. For graphene with one side exposed to air and one to hBN, $\epsilon_{1}=1$ and $\epsilon_{2} \simeq 4.5$. The value of $\epsilon_{2}$ has been taken from Ref. [8].

A good starting point to calculate plasmons in electron liquids is the so-called random phase approximation [25] (RPA) in which the full density-density response function $\chi_{\boldsymbol{G}, \boldsymbol{G}^{\prime}}(\boldsymbol{q}, \omega)$ in Eq. (4) is approximated by the solution of the following Dyson's equation:

$$
\begin{aligned}
\chi_{\boldsymbol{G}, \boldsymbol{G}^{\prime}}(\boldsymbol{q}, \omega)= & \chi_{\boldsymbol{G}, \boldsymbol{G}^{\prime}}^{(0)}(\boldsymbol{q}, \omega) \\
& +\sum_{\boldsymbol{G}^{\prime \prime}} \chi_{\boldsymbol{G}, \boldsymbol{G}^{\prime \prime}}^{(0)}(\boldsymbol{q}, \omega) v_{\boldsymbol{G}^{\prime \prime}}(\boldsymbol{q}) \chi_{\boldsymbol{G}^{\prime \prime}, \boldsymbol{G}^{\prime}}(\boldsymbol{q}, \omega),
\end{aligned}
$$

where $\chi_{\boldsymbol{G}, \boldsymbol{G}^{\prime}}^{(0)}(\boldsymbol{q}, \omega)$ is the density response function of the noninteracting electron system in the moiré superlattice. Off-diagonal terms with respect to RLVs in Eq. (5) represent crystal local field effects, which we retain since they may be important in comparing theory with experimental results [29]. sion:

The quantity $\chi_{\boldsymbol{G}, \boldsymbol{G}^{\prime}}^{(0)}(\boldsymbol{q}, \omega)$ is given by the following expres-

$$
\begin{aligned}
\chi_{\boldsymbol{G}, \boldsymbol{G}^{\prime}}^{(0)}(\boldsymbol{q}, \omega)= & \frac{2}{L^{2}} \sum_{\boldsymbol{k}, n ; \boldsymbol{k}^{\prime}, n^{\prime} ; \nu} \frac{n_{\mathrm{F}}\left(\varepsilon_{\boldsymbol{k}, n, v}\right)-n_{\mathrm{F}}\left(\varepsilon_{\boldsymbol{k}^{\prime}, n^{\prime}, v}\right)}{\hbar \omega+\varepsilon_{\boldsymbol{k}, n, v}-\varepsilon_{\boldsymbol{k}^{\prime}, n^{\prime}, v}+i \eta} \\
& \times \mathcal{M}_{\boldsymbol{k}, n, v ; \boldsymbol{k}^{\prime}, n^{\prime}, v}(\boldsymbol{q}+\boldsymbol{G}) \mathcal{M}_{\boldsymbol{k}, n, v ; \boldsymbol{k}^{\prime}, n^{\prime}, v}^{\dagger}\left(\boldsymbol{q}+\boldsymbol{G}^{\prime}\right),
\end{aligned}
$$

where the factor 2 accounts for spin degeneracy, $\eta$ is a positive infinitesimal, and $n_{\mathrm{F}}(x)=\left\{\exp \left[(x-\mu) / k_{\mathrm{B}} T\right]+\right.$ $1\}^{-1}$ is the Fermi-Dirac occupation factor at temperature $T$ and chemical potential $\mu$. Finally, $\mathcal{M}_{\boldsymbol{k}, n, v ; \boldsymbol{k}^{\prime}, n^{\prime}, v}(\boldsymbol{q}+\boldsymbol{G}) \equiv$ $\left\langle\boldsymbol{k}, n, \nu\left|e^{-i(\boldsymbol{q}+\boldsymbol{G}) \cdot \boldsymbol{r}}\right| \boldsymbol{k}^{\prime}, n^{\prime}, v\right\rangle$. We emphasize that Eq. (6) is the sum of two contributions, one for each principal valley $v=K, K^{\prime}$.

Self-sustained oscillations of an electron system in a crystal can be found by solving the equation $\operatorname{det}\left\{[1 / \chi]_{\boldsymbol{G}, \boldsymbol{G}^{\prime}}(\boldsymbol{q}, \omega)\right\}=0$ [25]. Alternatively, one can directly calculate the loss function $L(\boldsymbol{q}, \omega) \equiv-\operatorname{Im}\left\{[1 / \epsilon]_{\mathbf{0}, \mathbf{0}}(\boldsymbol{q}, \omega)\right\}$, which is appealing since it is directly measured by electron-energy-loss spectroscopy [30]. The loss function displays sharp peaks at the plasmon poles and carries also precious information on interband transitions and Landau damping. The latter determines the width of the plasmon peak in $L(\boldsymbol{q}, \omega)$. In this Rapid Communication we focus our attention on $L(\boldsymbol{q}, \omega)$.

Numerical results and discussion. A summary of our main results for the RPA loss function $L(\boldsymbol{q}, \omega)$-calculated at $T=10 \mathrm{~K}$ and for the illustrative moiré miniband structures in Figs. 2(a) and 2(b) -is reported in Figs. 3-5. All the results shown in Figs. 3-5 have been obtained for a wave vector $\boldsymbol{q}$

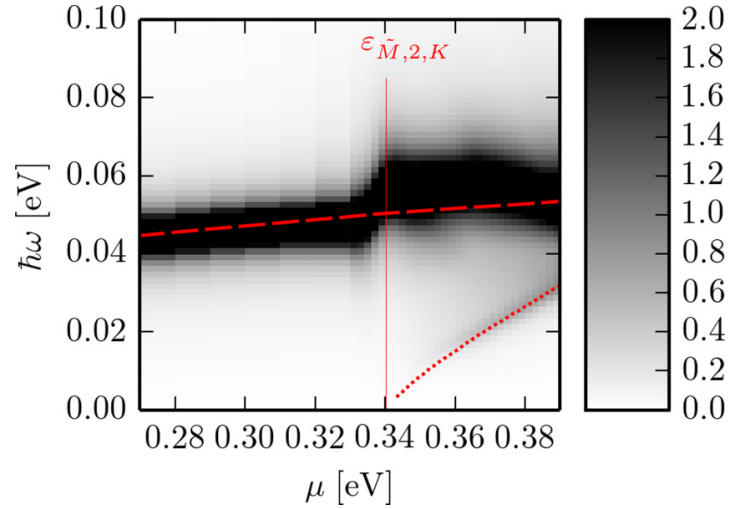

FIG. 3. (Color online) A 2D density plot of the RPA loss function $L(\boldsymbol{q}, \omega)$ for the superlattice miniband structure in Fig. 2(a) and $|\boldsymbol{q}|=0.007 \mathrm{~nm}^{-1}$. The vertical thin solid line denotes the bottom edge $\varepsilon_{\tilde{M}, 2, K}$ of the $n=2$ conduction miniband-see Fig. 2(a). The long-dashed line represents the chemical potential dependence of a DP with a Fermi velocity $v_{\mathrm{F}}$ equal to the bare value $v_{\mathrm{F}} \sim 10^{6} \mathrm{~m} / \mathrm{s}$. For chemical potentials above $\varepsilon_{\tilde{M}, 2, K}$, a satellite $\tilde{M}$-point plasmon is generated: Its chemical potential dependence (dotted line) follows that of a plasmon in a 2D parabolic-band electron gas. $1 \mathrm{D}$ cuts of this $2 \mathrm{D}$ density plot for different values of the chemical potential $\mu$ are reported in Ref. [28].

oriented along the $\tilde{\Gamma}-\tilde{M}$ direction. In the range of parameters explored in this work we have not noticed significant angular anisotropies of the satellite plasmons. (This has been checked by performing numerical calculations with $\boldsymbol{q}$ oriented along the $\tilde{\Gamma}-\tilde{K}$ and $\tilde{\Gamma}-\tilde{K}^{\prime}$ directions [28].) Technical details relative to the numerical approach have been reported in Ref. [28].

In Fig. 3 we plot the loss function $L(\boldsymbol{q}, \omega)$ for the superlattice miniband structure shown in Fig. 2(a). For chemical potentials below the bottom edge $\varepsilon_{\tilde{M}, 2, K}$ of the second conduction miniband, the loss function peaks at the usual DP mode, i.e., $\tilde{\Gamma}$-point plasmon (dashed line). For chemical potentials above $\varepsilon_{\tilde{M}, 2, K}$, we clearly see that a new satellite plasmon mode is generated at the $\tilde{M}$ point. Interestingly, the dotted line in Fig. 3, which tracks the chemical potential dependence of the

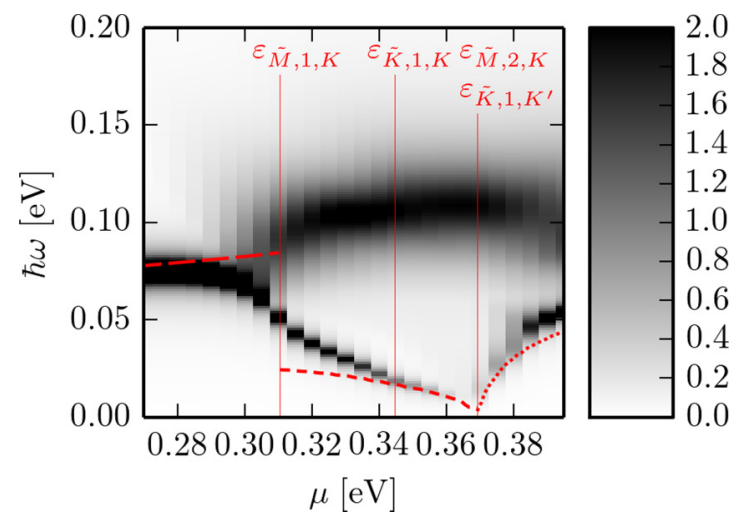

FIG. 4. (Color online) Same as in Fig. 3 but for the miniband structure shown in Fig. 2(c) and $|\boldsymbol{q}|=0.021 \mathrm{~nm}^{-1}$. The long-dashed and dotted lines have the same physical meaning as in Fig. 3. The short-dashed line represents the chemical potential dependence of a $\tilde{K}$-point plasmon stemming from a satellite Dirac point. Note that $\varepsilon_{\tilde{K}, 1, K^{\prime}}$ coincides with $\varepsilon_{\tilde{M}, 2, K}$. 


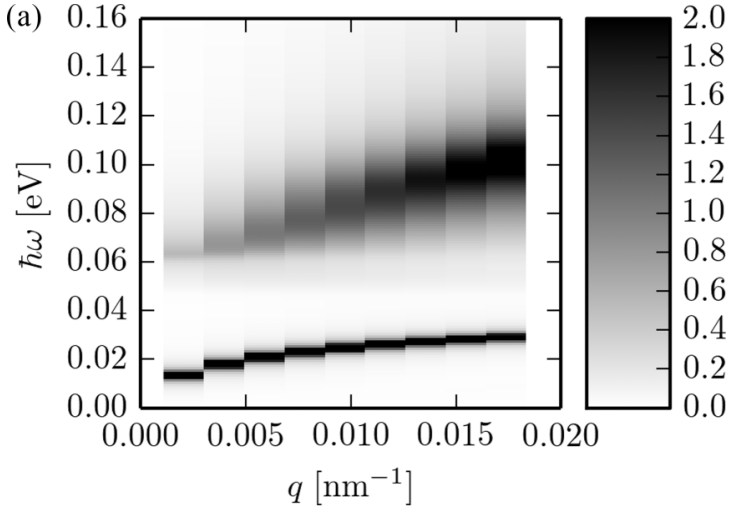

(b)

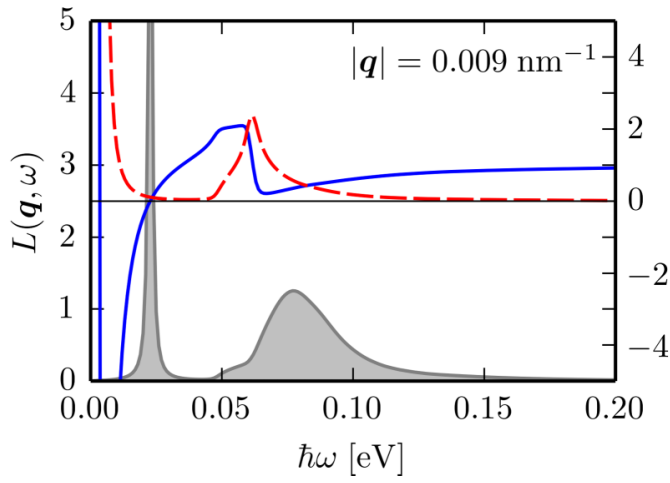

FIG. 5. (Color online) Wave vector and energy dependence of the loss function $L(\boldsymbol{q}, \omega)$ for $\mu=330 \mathrm{meV}$. All the other parameters are as in Fig. 4. In this plot $L(\boldsymbol{q}, \omega)$ has been evaluated for nine values of $q$. The gray-shaded area in (b) illustrates the energy dependence of $L(\boldsymbol{q}, \omega)$ for $q=0.009 \mathrm{~nm}^{-1}$. Solid and dashed curves represent $\operatorname{Re}\left[\epsilon_{\mathbf{0}, \mathbf{0}}(\boldsymbol{q}, \omega)\right]$ and $\operatorname{Im}\left[\epsilon_{\mathbf{0}, \mathbf{0}}(\boldsymbol{q}, \omega)\right]$, respectively. The values of these functions can be inferred from the vertical axis on the right. While the sharp peak at $\hbar \omega \simeq 20 \mathrm{meV}$ corresponds to a true zero of the macroscopic dielectric function $\epsilon_{\mathbf{0}, \mathbf{0}}(\boldsymbol{q}, \omega)$, the broad peak at $\hbar \omega \simeq$ $100 \mathrm{meV}$ does not.

new $\tilde{M}$-point plasmon, corresponds to the analytical formula of a plasmon excitation in a 2D parabolic-band electron gas [25], i.e., $\omega_{2 \mathrm{DEG}}^{2}=q \times 2 \pi n_{\tilde{M}, K}(\mu) e^{2} /\left(m^{\star} \epsilon\right)$, with band mass $m^{\star} \simeq 0.01 m_{\mathrm{e}}, m_{\mathrm{e}}$ being electron's mass in vacuum. Here, the quantity $n_{\kappa, v}(\mu)$ represents the density of a pocket of electrons or holes at the high-symmetry point $\kappa$ of the SBZ, in the valley $v=K, K^{\prime}$, and for a chemical potential $\mu$. The dependence of $n_{\kappa, v}$ on $\mu$ at $T=0$ is discussed in Ref. [28]. The parabolic-band-like (i.e., such as $n_{\tilde{M}, K}^{1 / 2}$ rather than $n_{\tilde{M}, K}^{1 / 4}$, as expected for a DP $[17,18])$ density dependence of this satellite mode is attributed to the parabolic dependence of the superlattice minibands on $\boldsymbol{k}$ near $\varepsilon_{\tilde{M}, 2, K}$ and for $\boldsymbol{k}$ along the $\tilde{M}-\tilde{K}$ direction-see Fig. 2(a).
The situation is even richer in the case $V_{\mathrm{s}}=0$ and $V_{\Delta} \neq 0$. Representative results are shown in Fig. 4, where we show the loss function $L(\boldsymbol{q}, \omega)$ for the miniband structure in Fig. 2(c). When increasing the chemical potential, the ordinary $\tilde{\Gamma}$-point DP morphs into a $\tilde{K}$-point DP with lower energy. Figure 5(a) shows that this mode displays a 2D dispersion $\propto \sqrt{q}$. Moreover, its chemical potential dependence is consistent with that of DPs [17], i.e., $\omega_{\mathrm{DP}}^{2}=q \times 2 \sqrt{\pi} v_{\mathrm{F}, \tilde{K}, K^{\prime}} n_{\tilde{K}, K^{\prime}}^{1 / 2}(\mu) e^{2} /(\hbar \epsilon)$ with an effective Fermi velocity $v_{\mathrm{F}, \tilde{K}, K^{\prime}} \simeq 0.3 v_{\mathrm{F}}$, which is reduced with respect to the Fermi velocity $v_{\mathrm{F}}$ in an isolated graphene sheet. Satellite Dirac points in the superlattice miniband structure enable $\tilde{K}$-point DP modes with low energy for dopings $\gtrsim 350 \mathrm{meV}$. Long-wavelength graphene superlattices therefore give access to long-lived, low-energy, e.g., terahertz, plasmons, which are difficult to reach due to the ultralow dopings $(\simeq 10 \mathrm{meV})$ that these modes require in the absence of a superlattice $[17,18]$ (ultralow carrier densities imply strong susceptibility to disorder and, in turn, short plasmon lifetimes).

A further increase in $\mu$ generates a satellite $\tilde{M}$-point plasmon similar to that in Fig. 3, with the same effective mass $m^{\star} \simeq 0.01 m_{\mathrm{e}}$. In Fig. 4 we also notice a broad peak at energy $\hbar \omega \simeq 100 \mathrm{meV}$. As demonstrated in Fig. 5(b), this peak is due to interband electron-hole excitations, which are quite bunched in energy. Indeed, the real part of the macroscopic dielectric function $\epsilon_{\mathbf{0}, \mathbf{0}}(\boldsymbol{q}, \omega)$ does not vanish for $\hbar \omega \simeq 100 \mathrm{meV}$ and $\operatorname{Im}\left[\epsilon_{\mathbf{0}, \mathbf{0}}(\boldsymbol{q}, \omega)\right]$ is large at the same energy.

Recently, it has been shown $[31,32]$ that graphene on $\mathrm{hBN}$ can display topologically nontrivial bands (i.e., bands yielding finite Chern numbers) in the case of commensurate stackings. It will be interesting to study the plasmonic properties of these special stacks, especially in a magnetic field. Due to the superb electronic quality of graphene on $\mathrm{hBN}$, the plasmon modes described above are characterized by very low damping rates $[23,24]$. We truly hope that our predictions will stimulate scattering-type near-field optical $[21,22]$ and electron-energyloss [30] spectroscopy studies of the plasmonic properties of graphene-hBN stacks.

Acknowledgments. It is a pleasure to thank Frank Koppens and Francesco Pellegrino for useful discussions. This work was supported by the European Community under Graphene Flagship (Contract No. CNECT-ICT-604391), MIUR (Italy) through the programs "FIRB-Futuro in Ricerca 2010"-Project PLASMOGRAPH (Grant No. RBFR10M5BT) and "Progetto Premiale 2012"-Project ABNANOTECH, MINECO (Spain) through Grant No. FIS2011-23713, and the European Research Council Advanced Grant (Contract No. 290846). We have made use of free software (http://www.gnu.org, http://www.python.org).
[1] K. S. Novoselov and A. H. Castro Neto, Phys. Scr., T 146, 014006 (2012).

[2] F. Bonaccorso, A. Lombardo, T. Hasan, Z. Sun, L. Colombo, and A. C. Ferrari, Mater. Today 15, 564 (2012).
[3] A. K. Geim and I. V. Grigorieva, Nature (London) 499, 419 (2013).

[4] C. R. Dean, A. F. Young, I. Meric, C. Lee, L. Wang, S. Sorgenfrei, K. Watanabe, T. Taniguchi, P. Kim, K. L. Shepard, and J. Hone, Nat. Nanotechnol. 5, 722 (2010). 
[5] L. Britnell, R. V. Gorbachev, R. Jalil, B. D. Belle, F. Schedin, A. Mishchenko, T. Georgiou, M. I. Katsnelson, L. Eaves, S. V. Morozov, N. M. R. Peres, J. Leist, A. K. Geim, K. S. Novoselov, and L. A. Ponomarenko, Science 335, 947 (2012).

[6] D. C. Elias, R. V. Gorbachev, A. S. Mayorov, S. V. Morozov, A. A. Zhukov, P. Blake, L. A. Ponomarenko, I. V. Grigorieva, K. S. Novoselov, F. Guinea, and A. K. Geim, Nat. Phys. 7, 701 (2011).

[7] R. V. Gorbachev, A. K. Geim, M. I. Katsnelson, K. S. Novoselov, T. Tudorovskiy, I. V. Grigorieva, A. H. MacDonald, S. V. Morozov, K. Watanabe, T. Taniguchi, and L. A. Ponomarenko, Nat. Phys. 8, 896 (2012).

[8] G. L. Yu, R. Jalil, B. Belle, A. S. Mayorov, P. Blake, F. Schedin, S. V. Morozov, L. A. Ponomarenko, F. Chiappini, S. Wiedmann, U. Zeitler, M. I. Katsnelson, A. K. Geim, K. S. Novoselov, and D. C. Elias, Proc. Natl. Acad. Sci. USA 110, 3282 (2013).

[9] M. Yankowitz, J. Xue, D. Cormode, J. D. Sanchez-Yamagishi, K. Watanabe, T. Taniguchi, P. Jarillo-Herrero, P. Jacquod, and B. J. LeRoy, Nat. Phys. 8, 382 (2012).

[10] L. A. Ponomarenko, R. V. Gorbachev, G. L. Yu, D. C. Elias, R. Jalil, A. A. Patel, A. Mishchenko, A. S. Mayorov, C. R. Woods, J. R. Wallbank, M. Mucha-Kruczynski, B. A. Piot, M. Potemski, I. V. Grigorieva, K. S. Novoselov, F. Guinea, V. I. Fal'ko, and A. K. Geim, Nature (London) 497, 594 (2013).

[11] C. R. Dean, L. Wang, P. Maher, C. Forsythe, F. Ghahari, Y. Gao, J. Katoch, M. Ishigami, P. Moon, M. Koshino, T. Taniguchi, K. Watanabe, K. L. Shepard, J. Hone, and P. Kim, Nature (London) 497, 598 (2013).

[12] B. Hunt, J. D. Sanchez-Yamagishi, A. F. Young, M. Yankowitz, B. J. LeRoy, K. Watanabe, T. Taniguchi, P. Moon, M. Koshino, P. Jarillo-Herrero, and R. C. Ashoori, Science 340, 1427 (2013).

[13] J. Xue, J. Sanchez-Yamagishi, D. Bulmash, P. Jacquod, A. Deshpande, K. Watanabe, T. Taniguchi, P. Jarillo-Herrero, and B. J. LeRoy, Nat. Mater. 10, 282 (2011).

[14] R. Decker, Y. Wang, V. W. Brar, W. Regan, H.-Z. Tsai, Q. Wu, W. Gannett, A. Zettl, and M. F. Crommie, Nano Lett. 11, 2291 (2011).

[15] See, for example, C.-H. Park, L. Yang, Y. W. Son, M. L. Cohen, and S. G. Louie, Nat. Phys. 4, 213 (2008); Phys. Rev. Lett. 101, 126804 (2008); Y. P. Bliokh, V. Freilikher, S. Savel'ev, and F. Nori, Phys. Rev. B 79, 075123 (2009); R. P. Tiwari and D. Stroud, ibid. 79, 205435 (2009); M. Barbier, P. Vasilopoulos, and F. M. Peeters, ibid. 81, 075438 (2010); R. Bistritzer and A. H. MacDonald, ibid. 84, 035440 (2011); Proc. Natl. Acad. Sci. USA 108, 12233 (2011); P. Burset, A. L. Yeyati, L. Brey, and H. A. Fertig, Phys. Rev. B 83, 195434 (2011); S. Wu, M. Killi, and A. Paramekanti, ibid. 85, 195404 (2012); C. Ortix, L. Yang, and J. van den Brink, ibid. 86, 081405 (2012); M. Zarenia, O. Leenaerts, B. Partoens, and F. M. Peeters, ibid. 86, 085451 (2012); M. M. Kindermann, B. Uchoa, and D. L. Miller, ibid. 86, 115415 (2012); J. Jung, A. Raoux, Z. Qiao, and A. H. MacDonald, ibid. 89, 205414 (2014); J. Jung, A. DaSilva, A. H. MacDonald, and S. Adam, arXiv:1403.0496.
[16] J. R. Wallbank, A. A. Patel, M. Mucha-Kruczyński, A. K. Geim, and V. I. Fal'ko, Phys. Rev. B 87, 245408 (2013).

[17] F. H. L. Koppens, D. E. Chang, and F. J. García de Abajo, Nano Lett. 11, 3370 (2011); A. N. Grigorenko, M. Polini, and K. S. Novoselov, Nat. Photonics 6, 749 (2012); T. Stauber, J. Phys.: Condens. Matter 26, 123201 (2014); T. Low and P. Avouris, ACS Nano 8, 1086 (2014); F. J. García de Abajo, ACS Photonics 1, 135 (2014).

[18] See, for example, B. Wunsch, T. Stauber, F. Sols, and F. Guinea, New J. Phys. 8, 318 (2006); E. H. Hwang and S. Das Sarma, Phys. Rev. B 75, 205418 (2007); M. Polini, R. Asgari, G. Borghi, Y. Barlas, T. Pereg-Barnea, and A. H. MacDonald, ibid. 77, 081411(R) (2008); A. Principi, M. Polini, and G. Vignale, ibid. 80, 075418 (2009).

[19] M. Freitag, T. Low, W. Zhu, H. Yan, F. Xia, and P. Avouris, Nat. Commun. 4, 1951 (2013).

[20] L. Vicarelli, M. S. Vitiello, D. Coquillat, A. Lombardo, A. C. Ferrari, W. Knap, M. Polini, V. Pellegrini, and A. Tredicucci, Nat. Mater. 11, 865 (2012); D. Spirito, D. Coquillat, S. L. De Bonis, A. Lombardo, M. Bruna, A. C. Ferrari, V. Pellegrini, A. Tredicucci, W. Knap, and M. S. Vitiello, Appl. Phys. Lett. 104, 061111 (2014).

[21] Z. Fei, A. S. Rodin, G. O. Andreev, W. Bao, A. S. McLeod, M Wagner, L. M. Zhang, Z. Zhao, M. Thiemens, G. Dominguez, M. M. Fogler, A. H. Castro Neto, C. N. Lau, F. Keilmann, and D. N. Basov, Nature (London) 487, 82 (2012).

[22] J. Chen, M. Badioli, P. Alonso-González, S. Thongrattanasiri, F. Huth, J. Osmond, M. Spasenović, A. Centeno, A. Pesquera, P. Godignon, A. Zurutuza Elorza, N. Camara, F. J. García de Abajo, R. Hillenbrand, and F. H. L. Koppens, Nature (London) 487, 77 (2012).

[23] A. Principi, G. Vignale, M. Carrega, and M. Polini, Phys. Rev. B 88, 195405 (2013).

[24] A. Principi, G. Vignale, M. Carrega, and M. Polini, Phys. Rev. B 88, 121405(R) (2013).

[25] G. F. Giuliani and G. Vignale, Quantum Theory of the Electron Liquid (Cambridge University Press, Cambridge, UK, 2005).

[26] N. W. Ashcroft and N. D. Mermin, Solid State Physics (Saunders College Publishing, Fort Worth, TX, 1976).

[27] Following Ref. [16], we assume that inversion-asymmetric terms (which are allowed for graphene on $\mathrm{hBN}$ ) have negligible magnitude.

[28] See Supplemental Material at http://link.aps.org/supplemental/ 10.1103/PhysRevB.90.161406 for further details on the results and on the numerical implementation of the calculations.

[29] For the case of coinage metals, see, for example, A. Alkauskas, S. D. Schneider, C. Hébert, S. Sagmeister, and C. Draxl, Phys. Rev. B 88, 195124 (2013).

[30] R. F. Egerton, Rep. Prog. Phys. 72, 016502 (2009).

[31] J. C. W. Song, P. Samutpraphoot, and L. S. Levitov, arXiv:1404.4019.

[32] P. San-Jose, Á. Gutiérrez, M. Sturla, and F. Guinea, Phys. Rev. B 90, 115152 (2014). 